Authors

Robert K. Shelton, Long-Sheng Ma, Henry C. Kapteyn, Margaret M. Murnane, John L. Hall, and Jun Ye 
This article was downloaded by: [University of Colorado at Boulder Libraries]

On: 17 July 2015, At: 13:42

Publisher: Taylor \& Francis

Informa Ltd Registered in England and Wales Registered Number: 1072954 Registered office: 5 Howick Place, London, SW1P IWG

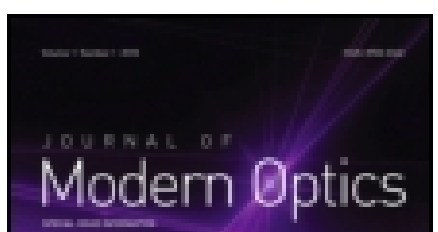

\section{J ournal of Modern Optics}

Publication details, including instructions for authors and subscription information:

http:// www.tandfonline.com/loi/ tmop20

\section{Active synchronization and carrier phase locking of two separate mode-locked femtosecond lasers}

ROBERT SHELTON ${ }^{a}$, LONG-SHENG MA ${ }^{a}$, HENRY KAPTEYN ${ }^{a}$, MARGARET MURNANE a , JOHN HALL ${ }^{a} \&$ JUN YE ${ }^{a}$

a JILA, National Institute of Standards and Technology and University of Colorado, Department of Physics, University of Colorado, Boulder, CO 80309-0440, USA Published online: 03 Dec 2010.

To cite this article: ROBERT SHELTON, LONG-SHENG MA, HENRY KAPTEYN, MARGARET MURNANE , J OHN HALL \& JUN YE (2002) Active synchronization and carrier phase locking of two separate mode-locked femtosecond lasers, J ournal of Modern Optics, 49:3-4, 401-409, DOI: 10.1080/09500340110088533

To link to this article: http:// dx. doi.org/ 10.1080/09500340110088533

\section{PLEASE SCROLL DOWN FOR ARTICLE}

Taylor \& Francis makes every effort to ensure the accuracy of all the information (the "Content") contained in the publications on our platform. However, Taylor \& Francis, our agents, and our licensors make no representations or warranties whatsoever as to the accuracy, completeness, or suitability for any purpose of the Content. Any opinions and views expressed in this publication are the opinions and views of the authors, and are not the views of or endorsed by Taylor \& Francis. The accuracy of the Content should not be relied upon and should be independently verified with primary sources of information. Taylor and Francis shall not be liable for any losses, actions, claims, proceedings, demands, costs, expenses, damages, and other liabilities whatsoever or howsoever caused arising directly or indirectly in connection with, in relation to or arising out of the use of the Content.

This article may be used for research, teaching, and private study purposes. Any substantial or systematic reproduction, redistribution, reselling, loan, sub-licensing, systematic supply, or distribution in any form to anyone is expressly forbidden. Terms \& Conditions of access and use can be found at http://www.tandfonline.com/page/terms-and-conditions 


\title{
Active synchronization and carrier phase locking of two separate mode-locked femtosecond lasers
}

\author{
ROBERT K. SHELTON, LONG-SHENG MA, \\ HENRY C. KAPTEYN, MARGARET M. MURNANE, \\ JOHN L. HALL and JUN YE \\ JILA, National Institute of Standards and Technology and University \\ of Colorado, and Department of Physics, University of Colorado, \\ Boulder, CO 80309-0440, USA
}

(Received Received 15 March 2001, revision received 5 May 2001)

\begin{abstract}
Two independent mode-locked femtosecond lasers are synchronized to an unprecedented precision. The rms timing jitter between the lasers is $4.3 \mathrm{fs}$ observed within a $160 \mathrm{~Hz}$ bandwidth over tens of seconds, or $26 \mathrm{fs}$ within a $50 \mathrm{kHz}$ bandwidth. Novel multi-stage phase-locked loops help to preserve this ultrahigh timing resolution while setting on arbitrary delay between the two pulse trains $(0-5 \mathrm{~ns})$. Under such synchronization, phase locking between the carrier frequencies of the two femtosecond lasers has been achieved. It is also demonstrated that the same level of synchronization can be achieved with two lasers at different repetition frequencies.
\end{abstract}

The ability to synchronize a passively mode-locked laser to an external reference, or to a second laser, has many applications in science and technology. Previous work in synchronizing two mode-locked Ti:sapphire lasers has demonstrated timing jitter of at best a few hundred femtoseconds (fs) [1, 2]. Since it is now routine to generate pulses with duration $<20 \mathrm{fs}$, improved techniques would make it possible to take full advantage of this time resolution for applications such as high power sum- and difference-frequency mixing, novel pulse generation and shaping, and for novel laser/accelerator based light sources [3]. Indeed, accurate timing of high intensity fields is essential for several important schemes in quantum coherent control and extreme nonlinear optics such as efficient X-ray generation [4]. Perfect synchronization between two ultrafast lasers would enable precision control of femtosecond lasers at the same level of technological maturity as for continuous-wave (cw) lasers, leading to unprecedented flexibility and bandwidth for pulse synthesis and optical comb generation.

A Kerr-lens mode-locked (KLM) femtosecond laser generates a regularly spaced comb of optical frequencies. Stabilization of this wide-bandwidth optical frequency comb has many useful applications [5, 6]. Precision phase control of the fs comb has already revolutionized the field of optical frequency metrology $[7,8]$, providing the capability of a direct phase-coherent link between optical and microwave frequencies. Frequency-domain control of fs combs not only provides an effective means to transfer the stability of a $\mathrm{cw}$ optical oscillator to the entire comb [9], but also has a strong impact on the time domain evolution of carrier-

Journal of Modern Optics ISSN 0950-0340 print/ISSN 1362-3044 online (C) 2002 Taylor \& Francis Ltd http://www.tandf.co.uk/journals DOI: $10.1080 / 09500340110088533$ 


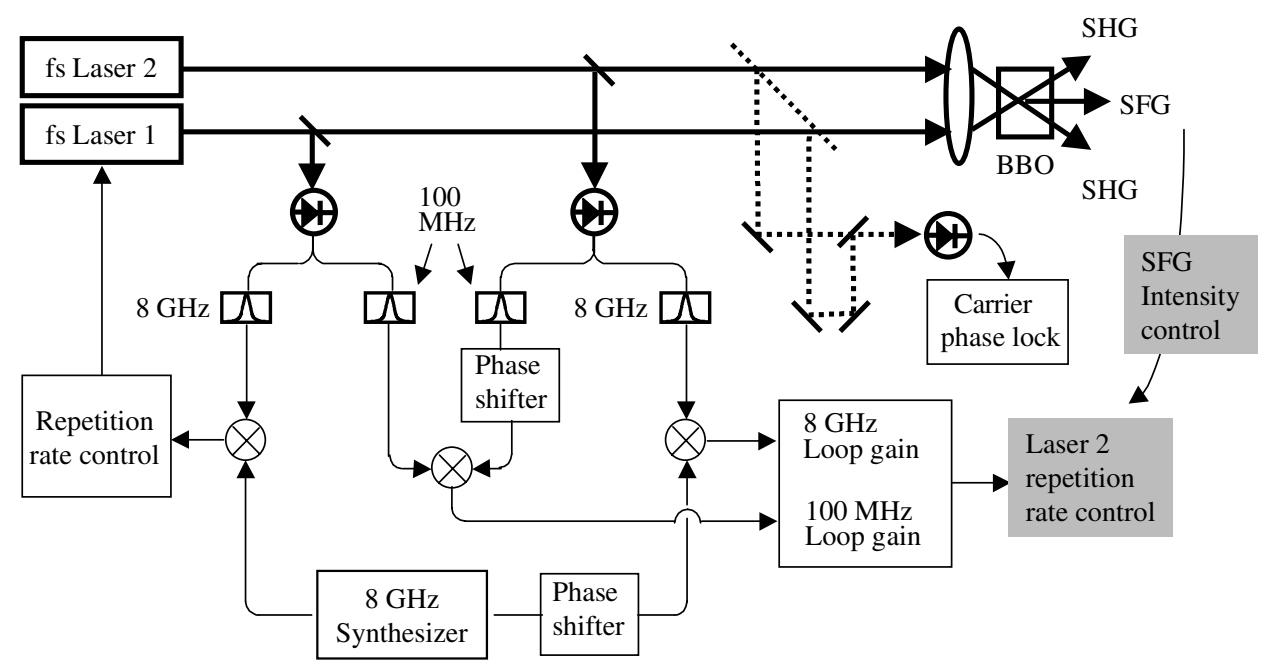

Figure 1. Experimental setup for timing synchronization of two fs lasers. The four phase-locked loops for synchronization are shown, along with the signal analysis scheme. Heterodyne detection of the carrier beat frequency between the two fs lasers is also shown.

envelope phase [10]. The frequency spacing of the comb interval, which represents one of the two degrees of freedom of the comb, has been controlled effectively either through a microwave clock $[7,8]$ or via a stable laser [9]. Since the frequency mode spacing of the fs comb is equal to the inverse of the cavity round trip time of the laser, reducing phase noise of the repetition rate spectrum directly improves the control of timing jitter.

In this paper, we demonstrate robust synchronization of pulse trains from two separate fs lasers, with a timing jitter of $<5 \mathrm{fs}$, at a bandwidth of $160 \mathrm{~Hz}$, observed over an interval of $1 \mathrm{~min}$. The two independent mode-locked Ti:sapphire lasers [11] operate at $780 \mathrm{~nm}$ and $820 \mathrm{~nm}$ respectively, with $\sim 100 \mathrm{MHz}$ repetition rates. One laser is pumped by a Spectra Physics Millenia and the other by a Coherent Verdi. The bandwidth of both lasers corresponds to a transform limit of $<20 \mathrm{fs}$ mode-locked pulses. The synchronization scheme shown in figure 1 employs two high-speed photodiodes to detect the two pulse trains. These signals are then input to four phase-locked loops (PLL) working at different timing resolutions. Using the first PLL, the repetition rate of laser 1 is synchronized to a stable RF source, with in-loop error signal indicating an rms timing jitter less than $10 \mathrm{fs}$ over a bandwidth of $10 \mathrm{kHz}$. Laser 2 is synchronized directly to laser 1 using the second PLL which compares and locks the fundamental frequencies of the two lasers at $100 \mathrm{MHz}$. The phase shift between the two $100 \mathrm{MHz}$ signals can be used to control the timing offset between two pulse trains. The third PLL compares the phase of the 80th harmonic of the two repetition frequencies; i.e. at $8 \mathrm{GHz}$. The third loop is activated and replaces the second one when the two pulse trains are nearly overlapped. This represents an electronic realization of a 'differential micrometer'-the $100 \mathrm{MHz}$ loop provides the full dynamic range of timing offset between two pulse trains, while the $8 \mathrm{GHz}$ loop produces enhanced phase stability of the repetition frequency. To minimize jitter further, the intensity of the sum frequency signal from the two pulse trains overlapped in space and time is directly 
(a)

(b)
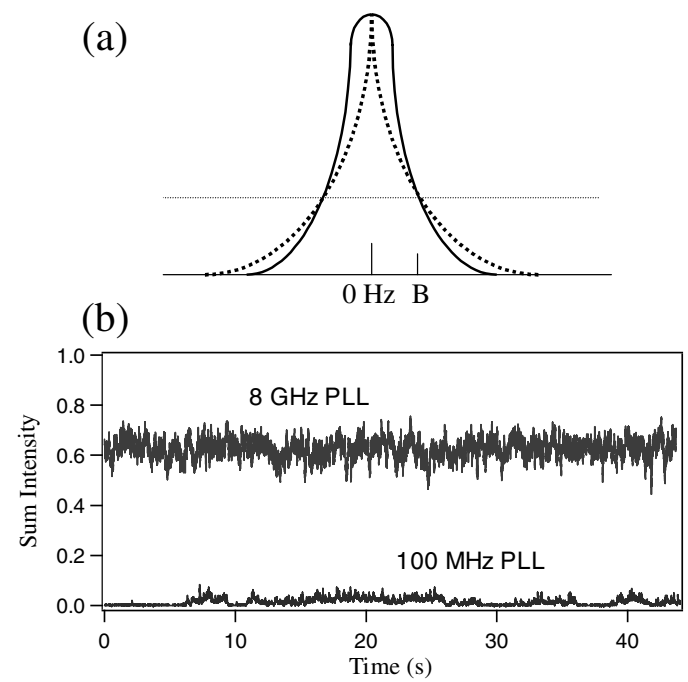

Figure 2. (a) Phase noise spectrum of the microwave reference signal ( $\cdots \cdots \cdot$ ) compared with the phase noise of the detected laser repetition signal $(-)$. At frequencies higher than $\mathrm{B}$, the laser has an intrinsically lower noise than the reference. $(b)$ Intensity signals of sum generation of the two fs lasers enabled by synchronization. The top trace represents the sum intensity for the $8 \mathrm{GHz}$ synchronization loop while the bottom trace corresponds to the $100 \mathrm{MHz}$ loop.

used as a control error signal for a fourth loop. This fourth loop can be usefully activated only when the $8 \mathrm{GHz}$ PLL is working effectively and most timing jitter noise has already been eliminated. All three loops actuate fast piezo-transducers (PZT) mounted on the laser end-mirrors. A combination of a fast, small, PZT and a slow, long, PZT is used to achieve a high servo bandwidth $(>50 \mathrm{kHz})$ and a large tuning range.

The motivation for using a high harmonic for phase locking the repetition rate is to obtain an enhanced signal-to-noise ratio $(S / N)$. In figure $2(a)$ the solid curve represents the detected phase noise power spectrum of the laser repetition signal, while the dotted curve indicates the microwave reference to which the repetition signal is locked. Experimental data show that the laser repetition signal has less fast phase noise than the $\mathrm{rf}$ reference beyond the frequency $\mathrm{B}$ (around $10 \mathrm{kHz}$ ) indicated in figure 2(a). Therefore the servo loop bandwidth should be limited to below $\mathrm{B}$, provided that the noise floor of the repetition detection is a few $\mathrm{dB}$ below the dashed line. If the detection $S / N$ is not sufficiently high, then the servo bandwidth will be further limited. Detection at a high harmonic can help to avoid this problem. The high-speed photodiodes used in this experiment suffer a loss of $S / N$ of only $3 \mathrm{~dB}$ when the repetition signal is detected at the 80 th harmonic, compared with the $S / N$ at the fundamental. When analysed at the fundamental while stabilized at the 80th harmonic, the repetition signal thus enjoys an effective increase in $S / N$ of $16 \mathrm{~dB}$.

Figure 3 shows the effective use of the combined PLLs for the control of the timing offset between two pulse trains. The second pulse train is scanned relative to the first via phase shift inside the $100 \mathrm{MHz}$ loop, showing the full range scanning capability in figure $3(a)$ and $(b)$. Once the two pulses are within $200 \mathrm{ps}$ of each other, we gradually reduce the gain of the $100 \mathrm{MHz}$ PLL and increase the 


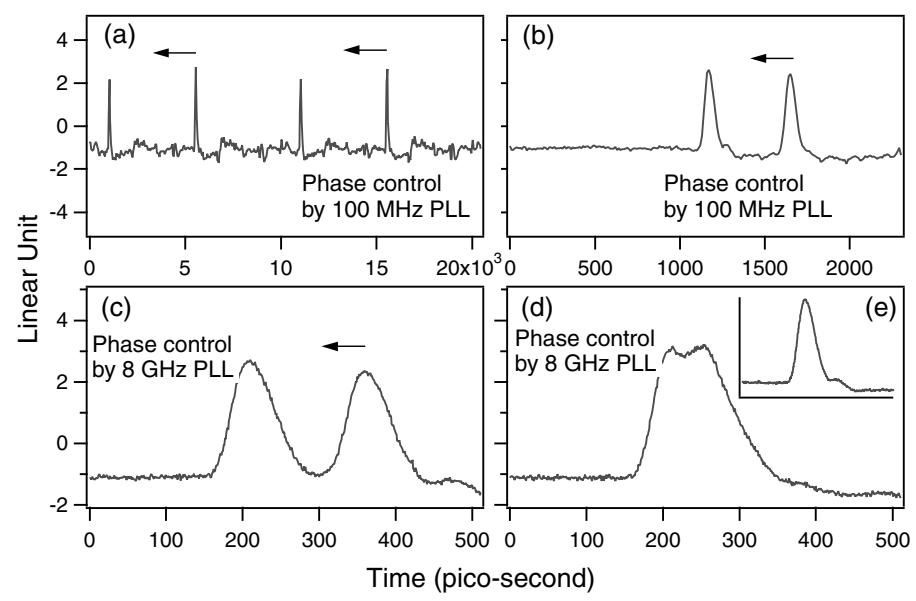

Figure 3. Control of the relative timing offset between the two independent pulse trains using the combined phase locked loops. Relative to laser 1, the pulse train from laser 2 is scanned via the rf phase shifter in the PLL. $(a)$ Two independent pulse trains incident on a same photodiode, with repetition period of $10 \mathrm{~ns}$. (b) Expanded time axis, $100 \mathrm{MHz}$ PLL control. (c) Time scale further expanded, $8 \mathrm{GHz}$ PLL control. (d) Two pulses nearly overlapped via adjustment of the $8 \mathrm{GHz}$ phase shifter. $(e)$ Two pulses maximally overlapped, permitting study of relative timing jitter.

gain of the $8 \mathrm{GHz}$ loop, leading to a smooth transition between the two loops and a small jump of the timing offset by at most $62.5 \mathrm{ps}$ ( $1 / 2$ of one $8 \mathrm{GHz}$ cycle). Tuning of the timing offset can then be continued with the $8 \mathrm{GHz}$ loop, as shown in figure $3(c)$ and $(d)$ with expanded time axis. The two pulses are maximally overlapped in figure $3(e)$.

To characterize our system further, the two pulse trains are focussed so that they cross in a $500-\mu \mathrm{m}$-thick, room temperature, $\beta$-Barium borate (BBO) crystal for nonlinear frequency generation (Type-I). Figure $4(a)$ shows the generated second harmonic (SHG) light from the two pulse trains after the crystal. When the two pulses are overlapped in space and time, sum frequency generation (SFG) is enabled, as shown with the bright spot in the centre of figure $4(b)$. We can thus use the intensity of the sum frequency generation as a diagnostic tool to study our system performance. Figure $2(b)$ shows the dramatic difference in the stability of sum frequency generation when the two lasers are synchronized with the $8 \mathrm{GHz}$ and the $100 \mathrm{MHz}$ loop. Figure $4(c)$ shows a cross-correlation measurement between the two pulses, carried out by a simple electronic sweep of the relative phase inside the $8 \mathrm{GHz}$ PLL. The Gaussian FWHM of the cross-correlation peak is $164 \mathrm{fs}$. This compares well with a theoretical estimate of $160 \mathrm{fs}$, based on the measured pulse widths of $82 \mathrm{fs}$ and $138 \mathrm{fs}$ for the individual lasers. (Because no extra-cavity dispersion compensation is used, the $20 \mathrm{fs}$ laser pulses are broadened due to dispersion.) Since the PLL system compares the phase of the two $8 \mathrm{GHz}$ signals with a double-balanced mixer, the phase scan can be accomplished by either tuning a phase shifter in the second signal path before the mixer or by summing a precision voltage waveform along with the output port of the mixer. Compared to a mechanical scanning system, this electronic tuning method offers a vastly superior performance in terms of the repeatability, reliability, and speed for 

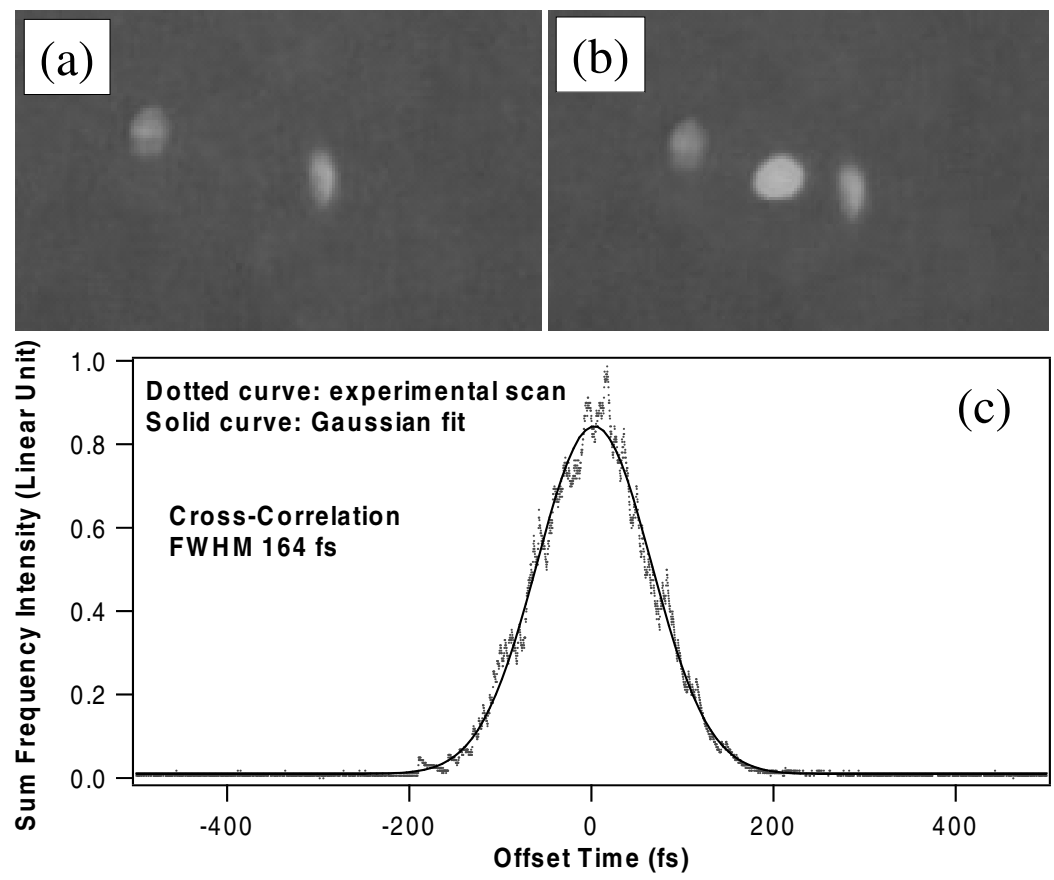

Figure 4. (a) Second harmonic generation from two spatially crossed pulse trains in a $\beta$-barium borate (BBO) crystal. (b) Sum generation is enabled when the two pulses are synchronized and overlapped. (c) Cross-correlation measurement via the electronic scan of relative phase.

setting the time offset, with no noticeable hysteresis $(<10 \mathrm{fs})$ when we switch back and forth between two pre-selected phase values.

The intensity fluctuations in the generated sum-frequency light are proportional to the timing jitter, particularly when the two pulses are offset in time by roughly half the pulse width. At short time scales, the intensity fluctuation of the sum frequency light can be studied by simply recording the pulse-to-pulse variation. Using a ns-speed photodiode and ns sampling rates, we directly digitized the sum frequency pulse train over a period of $10 \mu \mathrm{s}$. (The length of data is limited by the digitizer memory.) The recorded data is shown in figure $5(a)$, along with the expanded trace, when the relative timing offset between the two original pulse trains is near zero. The pulse-to-pulse variation of the sum frequency signal is basically the same as that of the original pulses from the two lasers-below a level of $0.4 \%$. Since the bandwidth of our servo loop does not quite reach the $10 \mu$ s time scale, this superior timing stability is attributed entirely to the free-running lasers owing to the lack of environmental disturbance on such short time scales. At longer time scales, the intensity fluctuation in the sum frequency signal can be explored with an appropriate low-pass filter to avoid sampling noise. A $50 \mathrm{kHz}$ low-pass filter is sufficient to suppress the discrete nature of the pulse train and permit the study of intensity fluctuations on a cw basis. In figure $5(b)$, the left trace shows the sum frequency intensity fluctuation when the two pulse trains are maximally overlapped, while the right trace is recorded when the two pulses are offset in time by half the pulse width. For the right trace, the sum frequency signal is monitored near the middle point of the cross-correlation peak shown in figure $4(c)$. Therefore 

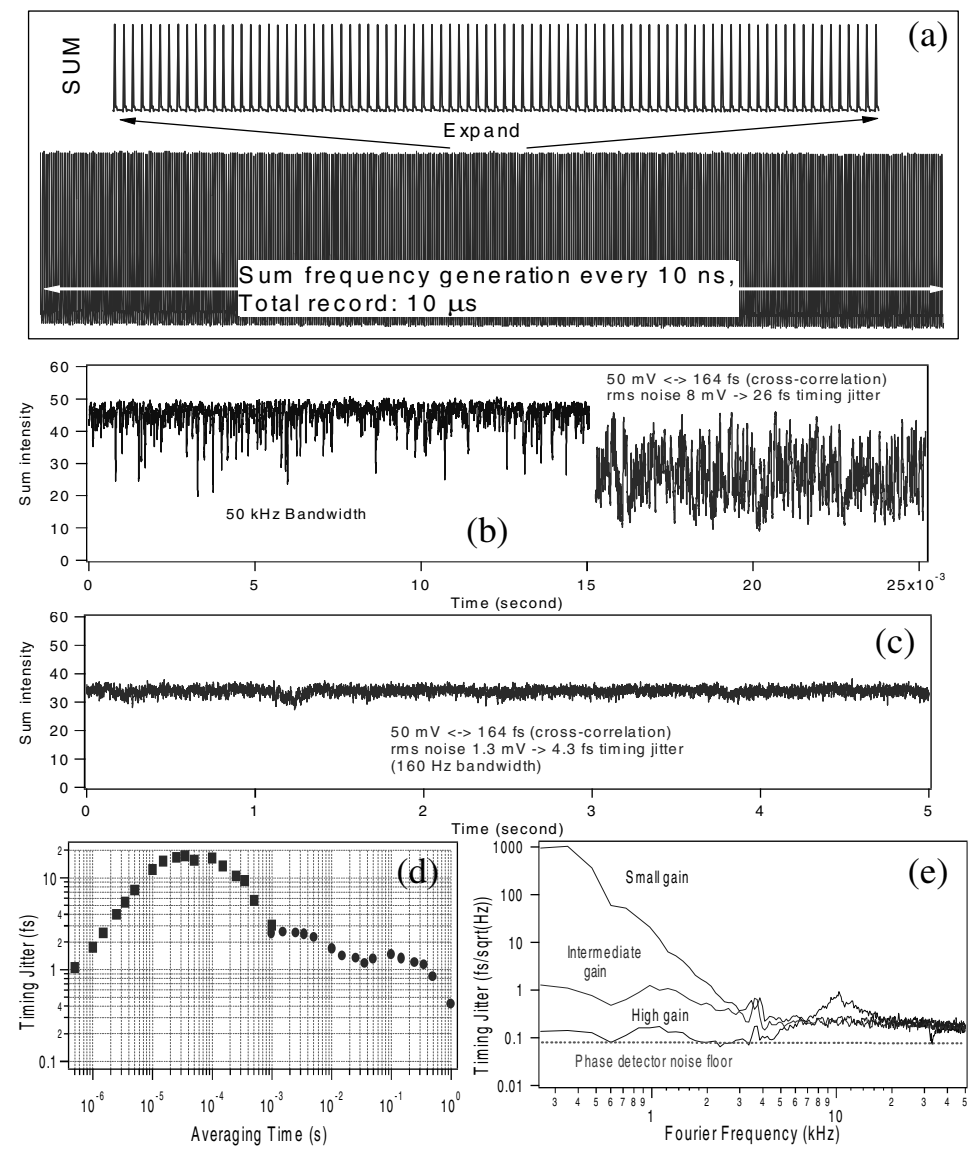

Figure 5. Timing jitter noise analysis. (a) A $10 \mu$ s direct digitization record of the sum frequency generation. Also shown is the time-expanded trace. The pulse-to-pulse intensity fluctuation is below $0.4 \%$, similar to the original laser intensity fluctuation. (b) Sum frequency generation observed with a $50 \mathrm{kHz}$ bandwidth. The left trace shows two pulses optimally overlapped and the right trace shows two pulses offset by a half width. (c) Sum signal observed under a $160 \mathrm{~Hz}$ bandwidth, showing an rms timing jitter noise of $4.3 \mathrm{fs}$. (d) Allan variation of the timing stability, showing the maximum jitter occurs around 10-100 $\mu$ s. (e) Fourier frequency spectrum of the error signal from the $8 \mathrm{GHz}$ phase-locked loop.

the intensity fluctuations can be translated linearly into timing jitter noise using the calibrated slope of the correlation peak. For a maximum intensity of the sum frequency light at 50 units, and the FWHM of the cross-correlation peak at $164 \mathrm{fs}$, we can conservatively estimate the conversion scale of the slope at $3.3 \mathrm{fs} /$ unit. The rms timing noise from the right trace is thus determined to be $\sim 26 \mathrm{fs}$ at a $50 \mathrm{kHz}$ bandwidth. At higher bandwidths, the jitter does not increase. If we use a bandwidth of $160 \mathrm{~Hz}$ (equivalent to $1 \mathrm{~ms}$ averaging time) the resultant intensity fluctuations of the summing light shown in figure $5(c)$ indicates rms timing jitter noise of $4.3 \mathrm{fs}$. We have recorded such stable performance over periods extending to nearly a minute. The advantage of the high harmonic PLL over the $100 \mathrm{MHz}$ loop is particularly clear from the recorded sum frequency intensity, which exhibits fluctuations more than 20 times bigger when under the $100 \mathrm{MHz}$ control. 
The synchronization lock can be maintained for durations of several hours, limited by the dynamic range of the servo PZT and by thermal expansion. A simple integrator stage can be implemented to control the temperature of the laser baseplate to extend the lock time even further.

It is interesting to explore further the issue of the timing jitter noise vs its characteristic time scales (or equivalently its frequency spectrum). Allan variance analysis [12] is a powerful technique developed for the study of frequency noise of stable oscillators in the time domain. The approach compares adjacent measurements segmented within certain time windows. In this first-difference calculation one is able to separate and isolate processes based on their time scales. This is useful to identify time scales at which fluctuations are the largest. From the time record of the timing jitter noise shown in figure $4(b)$ and $(c)$, we calculate the Allan variance of the timing jitter. The result is shown in figure $5(d)$. The data points [ $]$ correspond to the fast time record [the right trace in figure $5(b)$ ] while the data points $(\bigcirc)$ correspond to the longer record [shown in figure $5(c)$ ]. It is clear from this analysis that the dominant timing jitter noise occurs within 10-100 $\mu$ s, a region where the gain of the PLL loop is rolling off from the low frequency end while the external perturbations to the laser are increasing from the high frequency end. However, it is satisfying to find that the timing jitter noise never exceeds $20 \mathrm{fs}$ at any time scale.

These results can be confirmed through the equivalent analysis of the error signal within the PLL servo loop [13]. Figure $5(e)$ shows the Fourier spectrum analysis of the error signal at different gain settings. When the loop is operated stably with a high gain, within a frequency range from DC to $5 \mathrm{kHz}$ the error signal is very close to the limit $(\leq 0.1 \mathrm{fs} / \sqrt{\mathrm{Hz}})$ set by the intrinsic noise floor of the $8 \mathrm{GHz}$ mixer, i.e. the phase comparator used in the PLL. (We have removed the last two data points below $250 \mathrm{~Hz}$ that were affected by the DC offsets of the Fouriertransform spectrum analyser.) A small servo bump appears near $10 \mathrm{kHz}$ and rolls off to a flat noise floor near $0.14 \mathrm{fs} / \sqrt{\mathrm{Hz}}$. Integrating the noise density over the interested spectral range (from DC to $50 \mathrm{kHz}$ ) would yield an rms timing jitter noise of $\sim 30 \mathrm{fs}$, slightly larger than that indicated by the direct time domain data.

When the two lasers are well synchronized, heterodyne beat between the two corresponding sets of combs can be recovered with impressive $S / N$. Figure 1 shows the beat detection at another beam port independent from the synchronization work. A grating (1200 line/mm) is used to limit the optical bandwidth for the heterodyne beat. The beat detection effectively measures the difference in the carrier-envelope offset frequency between the two fs combs. Therefore by stabilizing the beat frequency to a mean value of $0 \mathrm{~Hz}$, the carrier-envelope phase evolution dynamics of one laser will be closely followed by the second laser. Locking of this beat frequency to $0 \mathrm{~Hz}$ can be conveniently implemented using an acousto-optic modulator (AOM). One laser beam passes through the AOM, picking up the AOM's frequency offset. The beat is then phase locked to the AOM's drive frequency, effectively removing the AOM frequency from the beat. Figure $6(a)$ illustrates the difference between the phase-locked and unlocked cases. When unlocked, the carrier beat frequency has a standard deviation of a few $\mathrm{MHz}$ with $1 \mathrm{~s}$ averaging time. Figure $6(b)$ shows the recorded beat frequency signal under phase locked condition. With an averaging time of $1 \mathrm{~s}$, the standard deviation of the beat signal is $1.3 \mathrm{~Hz}$. 

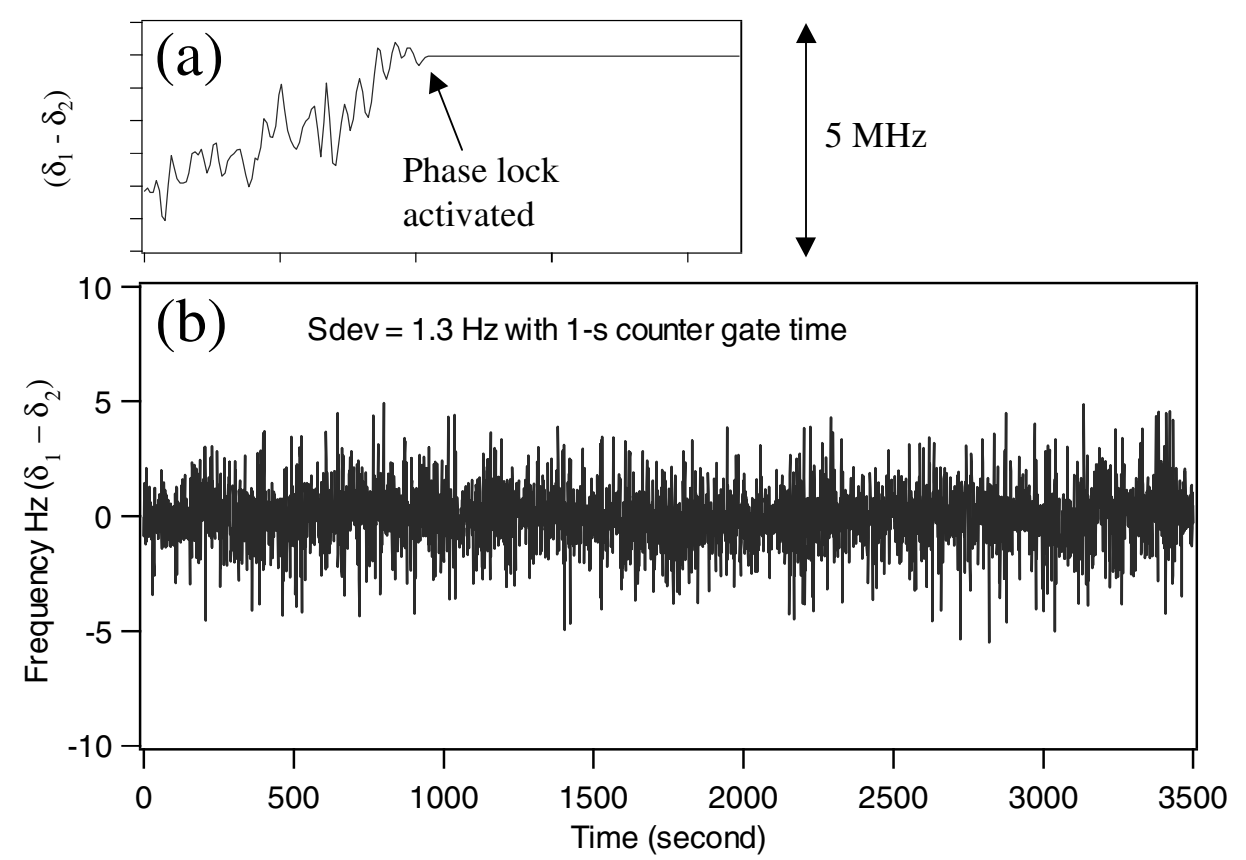

Figure 6. (a) Difference in the carrier frequency beat between phase-locked and unlocked cases. When unlocked, the standard deviation of the beat frequency is a few $\mathrm{MHz}$ at $1 \mathrm{~s}$ averaging time. (b) Record of the carrier beat signal between the two fs lasers when they are synchronized and phase locked. The beat frequency under the locked condition shows a standard deviation of $1.3 \mathrm{~Hz}$ at $1 \mathrm{~s}$ averaging time.

The flexibility of this synchronization system is demonstrated by locking two independent lasers working at different repetition frequencies. The 81 st harmonic of the repetition rate of laser 1 , still operating at $100 \mathrm{MHz}$, is compared to the 90th harmonic of laser 2, now running at $90 \mathrm{MHz}$. The two pulse trains will then collide in the time domain every $100 \mathrm{~ns}$, leading to a sum-frequency pulse train at a $10 \mathrm{MHz}$ repetition-rate. The result is shown in figure 7 , where the top trace reflects the summing frequency generation and the bottom trace is the second harmonic generation of the $100 \mathrm{MHz}$ laser. Notice the slight decrease in the second harmonic intensity when sum frequency generation occurs with two pulses overlapped. This approach can be generalized to produce sum- and differencefrequency generations with arbitrary repetition rates, yet perfectly synchronized to the master frequency.

In summary, we have synchronized two independent mode-locked femtosecond lasers to an unprecedented precision. The remaining rms timing jitter between the lasers is $4.3 \mathrm{fs}$ observed within a $160 \mathrm{~Hz}$ bandwidth over tens of seconds, or $26 \mathrm{fs}$ within a $50 \mathrm{kHz}$ bandwidth. This ultrahigh timing resolution is available through the entire dynamic range of pulse repetition period $(10 \mathrm{~ns})$. Such a degree of synchronization is the basis for realization of an optical carrier phaselocked loop between independent femtosecond lasers [14]. We have also demonstrated that such synchronization can be achieved with two lasers at different repetition frequencies. The techniques developed should prove to be invaluable in many research areas of ultrafast science and extreme nonlinear optics. 


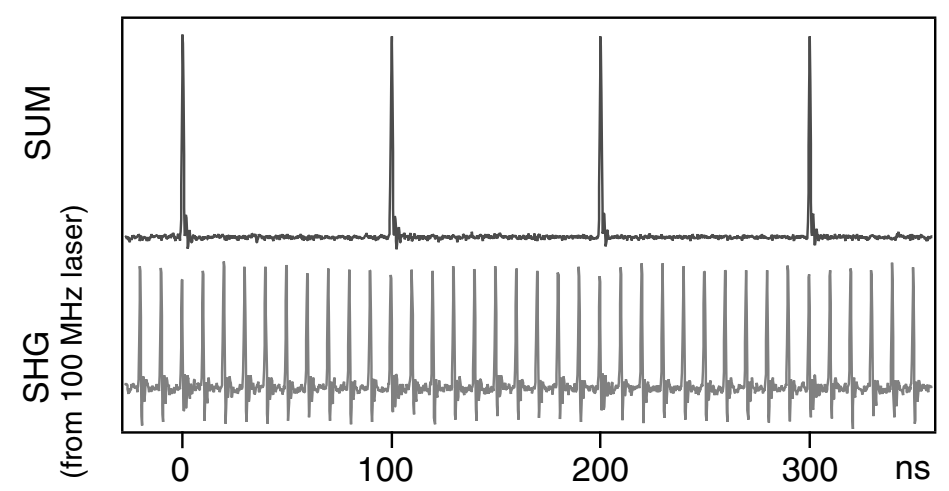

Figure 7. Sum frequency generation from two synchronized fs lasers that have different repetition rates, one at $90 \mathrm{MHz}$ and the other at $100 \mathrm{MHz}$. The second harmonic signal from the $100 \mathrm{MHz}$ laser is also shown. Notice the slight decrease in the second harmonic signal when sum frequency generation occurs with two pulses overlapped.

\section{Acknowledgments}

We are indebted to D. Anderson and KMLabs for the loan of pump lasers. We also thank S. T. Cundiff for stimulating discussions. The work at JILA is supported by the National Institute of Standards and Technology, NASA, National Science Foundation and the Research Corporation.

\section{References}

[1] Spence, D. E., Dudley, J. M., Lamb, K., Sleat, W. E., and Sibbett, W., 1994, Opt. Lett., 19, 481.

[2] Crooker, S. A., Betz, F. D., Levy, J., and Awschalom, D. D., 1996, Rev. Sci. Instru., 67, 2068.

[3] Schoenlein, R. W., Leemans, W. P., Chin, A. H., Volfbeyn, P., Glover, T. E., Balling, P., Zolotorev, M., Kim, K.-J., Chattopadhyay, S., and Shank, C. V., 1996, Science, 274, 236.

[4] Kobayashi, K., Miura, T., Ito, S., Zhang, Z. G., Torizuka, K., and Endo, A., 2000, Nucl. Instrum. Methods A, 455, 239.

[5] Teets, R. , Eckstein, J., and Hänsch, T. W., 1977, Phys. Rev. Lett., 38, 760.

[6] Baklanov, Y. V., and Chebotayev, V. P., 1977, Appl. Phys., 12, 97.

[7] Udem, Th., Reichert, J., Holzwarth, R., and Hänsch, T. W., 1999, Phys. Rev. Lett., 82, 3568; Udem, Th., Reichert, J., Holzwarth, R., and Hänsch, T. W., 1999, Opt. Lett., 24, 881.

[8] Diddams, S. A., Jones, D. J., Ye, J., Cundiff, S. T., Hall, J. L., Ranka, J. K., Windeler, R. S., Holzwarth, R., Udem, Th., and Hänsch, T. W., 2000, Phys. Rev. Lett., 84, 5102 .

[9] Ye, J., Hall, J. L., and Diddams, S. A., 2000, Opt. Lett., 25, 1675.

[10] Jones, D. J., Diddams, S. A., Ranka, J. K., Stentz, A., Windeler, R. S., Hall, J. L., and Cundiff, S. T., 2000, Science, 288, 635.

[11] Asaki, M. T., Huang, C.-P., Garvey, D., Zhou, J., Kapteyn, H. C., and Murnane, M. M., 1993, Opt. Lett., 18, 977.

[12] Allan, D. W., 1966, Proc. IEEE, 54, 221.

[13] Tsuchida, H., 1999, Opt. Lett., 24, 1641.

[14] Shelton, R. K., Ma, L.-S., Kapteyn, H. C., Murnane, M. M., Hall, J. L., and Ye. J. (to be published, 2001). 